\title{
From QFT to 2-d Supersymetric TQT:Mirror symmetry between Math and Physic
}

\author{
PHILIPPE DURAND \\ Conservatoire National des Arts et Métiers \\ Mathematics and Statistics Department \\ Laboratory M2N292 Rue Saint-Martin 75003 Paris \\ FRANCE
}

\begin{abstract}
Theoretical physics is taking an increasing part in the universe of mathematics. After calculus, vector and tensorial analysis, topological theories make their entry into quantum field theories. More precisely, in this domain, topological theories are the most relevant. A fundamental theorem of the Atiyah has important repercussions in several branches of quantum physics in the geometric approach. We can cite the work of Alain Connes on non-commutative geometry, but also all the developments due to Donaldson, E. Witten around Gauge theories, superstring and Mirror Symmetry. We present here an historical survey of some topological field theories, especially Mirror Symmetry to understand the interpenetration between quantum physics and topology.
\end{abstract}

Key-Words: Superstring theory, Supersymmetry, Mirror Symmetry, K-theory

Received: December 19, 2019. Revised: March 3, 2020. Accepted: March 14, 2020. Published: March 20, 2020.

\section{Introduction}

The introduction of supersymmetric theories into twodimensional field theories has helped to strengthen the links between geometry and physics. The formalism of string theory takes root around two-dimensional conformal fields theories, the special structure of the source manifold(Riemann surface) and supersymmetry which extends Noether symmetry by the addition of superymetric variables made it possible to extend the BRST formalism to make these theories topological. The computation of correlation functions in which points are replaced by classes of cohomologies, lead to define spaces of instantons (moduli spaces) whose dimension calculated by the index theorem can be made equal to zero by adding certain constraints and lead to staging enumerative geometry. At the same moment, Gromov and Witten [1] realize the importance of the concept of "curves" in symplectic geometry, A little later, a very elaborate form of Tduality, the mirror symmetry, played a decisive role to calculate some invariants of (Gromov Witten invariants) for the quintics treefold .The presentation will give some of the stages of this intellectual development and the relevance of the coupling between geometry and physics. From the tools that will be exposed in this paper, our goal is to identify the baground geometry and physics to understand the T-duality and mirror symmetry of models $A$ and $B$. The past recents years have seen the gradual fusion of geometry and physics. This program initiated at the start of the twentieth century by Einstein has not stopped growing since. There are several key period. The first between 1915 and 1930 sees the birth of Einstein's general relativity and that of the works of Oscar Kaluza and Felix Klein, the first attempt is to unify the fundamental interactions known at this moment: Relativity and electromagnetism. In addition returns to Klein the idea taken up by string theorists is then of rolled dimensions. Then quantum mechanics comes on the scene making us forgetting geometry a bit in favor of functional analysis and probabilities. Geometry comes back on the scenein the fifties with the notions of bundles and connections in differential geometry. The Yang-Mills theory, an example of a nonAbelian gauge theory modeled on the bundle $S U(2)$, comes into play. It prefigures the time of gauges theories in four dimension. Finally a third period begins at the start of the adaptation of supersymmetric models to string theory in the nineties . A first model of superstrings is born, Theory of superstrings of type I. it continues until now. Geometry is present everywhere; A string can be seen as a parameterized "complex" curve: a map of a Riemann surface in a Lorentzian manifold (bosonic strings). A superstring evolves in a richer space, a special complex manifold: called Calabi-Yau. Thus we can modelise the classical evolution of a string as the evolution of a point particle in space time. One of the big differences lies in the fact that an interval of time the source space of the classical theory must be replaced by a richer topolog- 
ically space namely a riemann surface. In the same way the target space is a particular Kahlerian manifold .That explains why the developments in algebraic geometry mainly due to Alexander Grothendieck at the beginning of the sixties, those of Atiyah about index theorem and developments $K$-topological theory, those of Kodaira and Spencer on the theory of deformations of complex manifold, those of Misha Gromov in the mid-eighties on the theory of holomorphic curves will play a major role in understanding this physical theory.

We will discuss a little bit about geometric modeling of string theory and especially mirror symmetry. String theory developed considerably thanks to the progress of complex geometry and algebraic geometry in the fifties during the Cartan seminar and then in the sixties thanks to Alexander Grothendieck. The beginning of the sixties sees the result of the demonstration of the Index theorem by Atiyah [2] which reconciles analysis and topology. This will later calculate the dimension of spaces, of modulus of curves. This theorem is in fact a smoother and more flexible version of the Riemann-Roch theorem [4] obtained in algebraic geometry. With the proof of the index theorem, we see the development of the $K$-topological theory extending algebraic topology to fiber bundles and which will be of the greatest importance in open string and branes theory to deal with the difficult question of topological defaults and hope to have an intrinsic and non-disturbing vision of the theory of everything. It should also be noted that these analytical-topological developments will be taken up in the symplectic version of string theory. Faced with the rigidity of the notion of holomorphic functions, Misha Gromov will enrich the landscape of complex and symplectic geometry by putting forward the notion of curves before that of functions. The genial idea comes back to being interested in the sections: holomorphic curves rather than in holomorphic functions, thus the landscape of geometry will be revisited through parametric curves (the parameters of evolution are found those of the classical string evolving in a space-time; in parallel algebraic geometry is developed and the theory of deformation born from Kodaira-Spencer's [5] work in complex geometry, derived functors enrich symplectic developments. Thus through derived functors algebraic geometers will provide complementary tools study of stable bundles on algebraic curves and enrich the tools from the $K$-theory for the study of branes.

\section{Quantum field theory}

\subsection{Field theory and symmetries}

The concept of field is fundamental in physics. A field $\varphi$ is a function of a source space (world sheet for string theory ) into a target space, $M$ with a sufficient number of dimensions. So this given a "package" $(\Sigma, M, \varphi)$ and a classical action: $S$ where: $\Sigma$ is the source space, often a manifold: for The classical mechanic of the point is the time axis (world line). For the conformal field theories like bosonic string theories: Riemann surface ...

Lagrangian density is a function on one or more fields and its first derivatives:

$$
\mathcal{L}=\mathcal{L}\left(\varphi_{1}, \varphi_{2}, \ldots, \partial_{\mu} \varphi_{1}, \partial_{\mu} \varphi_{2} \ldots\right)
$$

Classical action is the integral of the classical Lagrangian density on space $S=\int \mathcal{L} d^{n+1} x$

Principle of least action: The minimization of the action: $(\delta S=0$, leads to each field noted just $\varphi$ to the Euler-Lagrange equation which gives the equations of motion of the particle

$$
\frac{\partial \mathcal{L}}{\partial \varphi}-\partial_{\mu}\left(\frac{\partial \mathcal{L}}{\partial\left(\partial_{\mu} \varphi\right)}\right)=0
$$

\subsubsection{Noether Symmetries}

Symmetry of the action: the role of symmetry in physics is essential. We want, for example, such action invariant through a transformation like translation, rotation ...: if $\varphi \rightarrow \varphi+\delta \varphi$ alors $S \rightarrow S+\delta S$ Noether's theorem say: through any symmetry, the action is the same: $\delta S=0$ for example, in the case of the free particle, the symmetry by translation is equivalent to the conservation of momentum $p=m \dot{x}$

\subsection{From Field Theory to Quantum Field Theory}

A quantum field theory can be seen in through a classical action quantified from the path integral. We can then define the correlation functions dependent on selected observable. These functions depend, for example, the metric that is chosen on the target space.

\subsubsection{Quantum fields, QFT}

Uncertainty on the position or momentum in quantum mechanic led to replace the classical solution (least action) by the partition function or the set of all possible solutions: It is the path integral

$$
\mathcal{Z}=\int_{\Sigma \rightarrow M} e^{-S(\varphi)} \mathcal{D} \varphi
$$


Similarly, one can calculate correlation functions, or functions with $n$ marked points.

$$
\begin{array}{r}
<\varphi_{1}\left(x_{1}\right), \ldots, \varphi_{n}\left(x_{n}\right)>= \\
\int_{\Sigma \rightarrow M} \varphi_{1}\left(x_{1}\right) \ldots \varphi_{n}\left(x_{n}\right) e^{-S(\varphi)} \mathcal{D} \varphi
\end{array}
$$

The simplest example which is the leitmotif of the theory of renormalization is the two-dimensional model on lattice, is the Ising model: Here $\boldsymbol{\Sigma}$ is a lattice: $\bigwedge$ and $\mathbf{M}=+,-$

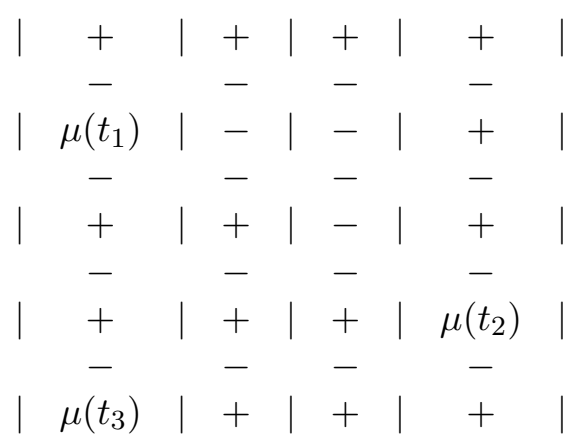

Figure 1: Ising model

The figure represents the Lattice and correlations between some marked points.

Here the action is: $S(\mu)=-\frac{\beta}{2 \pi} \sum_{|t-s|=1} \mu(t) \mu(s)$

Correlators are defined as mean values in some lattice points :

$$
\begin{array}{r}
<\mu\left(x_{1}\right), \mu\left(x_{2}\right), \ldots, \mu\left(x_{n}\right)>= \\
\frac{\Sigma_{M a p}(\Sigma, M)}{\mu\left(x_{1}\right) \ldots \mu\left(x_{n}\right) e^{-S(\mu)} \mathcal{D} \mu} \\
\Sigma_{M a p(\Sigma, M)} e^{-S(\mu)} \mathcal{D} \mu
\end{array}
$$

\section{Quantum field theory for strings}

The quantum field theory adapted to string theory is the conformal field theory [6]. this theory is very complex for the mathematician and it is not our purpose to describe it completely. Let us remember, however, that the evolution of a string can be described by a Riemann surface or complex curve. the adapted model is the sigma model, and particularly, the supersymmetric sigma model. In this model, the source space is a Riemann surface and for the moment, the target space supposed to be a complex variety.

\subsection{Moduli space of Riemann Surfaces}

In the extended field theories used for string theory, in quantum field theory, the word line that leads to the definition of Feymann diagrams is replaced by the world sheet: a Riemann surface. Riemann's theory of surfaces can nowadays be considered successful. We can see them from the angle of symplectic geometry where an important parameter is the area, or from the angle of complex geometry, the important parameter is then the complex structure $J$ adopted. We will see from the angle of mirror symmetry, these parameters are in duality. A whole mathematical engineering can then be carried out, leading to the definition of spaces of moduli of curves stabilized by the addition of marked points. These concepts have been well known since the work of Riemann and Roch. Conformal field theory uses these results to define the concept of sigma model, and the concepts of stable curves and stable maps generalize the Feynman diagrams and $n$-point correlation functions of quantum field theory.

\subsection{The Mathematics of Gromov and Grothendieck}

The global study of schemes or varieties is very difficult. Grothendieck already started studying moduli of curves, more malleable, as that means study object through its sections which is much easier. Gromov noted that. There are very few holomorphic functions on a complex manifold or almost-complex like symplectic manifolds.

The big idea of Gromov [7] is to consider the study of a symplectic manifold through its sections that are: holomorphic curves: In mathematics the worldsheet is a curve (Riemann surface), the evolution of a bosonic string that is a field of $\Sigma \rightarrow X$ is a parameterized curve. We can present the Gromov-Witten invariants by one of these two views Symplectic Geometry (Ruan, Tuan) [1], Algebraic Geometry (Pandaripande, Katz) [8].

\subsection{Symplectic geometry and pseudoholo- morphic curves}

Recall that a symplectic manifold is a differential manifold equipped with a 2-closed form $\omega:(M, \omega)$. The simplest example is $\mathbb{R}^{2 n}$ and $\omega=\sum d x_{i} \wedge d y_{i}$, We can also consider the space $\mathbb{C}^{n}$ or create new complex structures $J$ which are all integrable. Thus $\mathbb{R}^{2 n}$ can be endowed with a structure of simplectic manifold which is not always the case. A parameterized 
curve is a map: $\varphi:(\Sigma, j) \rightarrow(M, J)$, where $j$ is an almost complex structure on $\Sigma, J$ be an almost complex structure on the target. A parametrized curve is holomorphe if its differential verifies equations of CauchyRiemann: $J \circ d \varphi=d \varphi \circ j$, (the differential is $\mathbb{C}$ linear).

\subsection{Riemann-Roch formula moduli space of curves}

For now we place ourselves in the more general context of algebraic geometry. We recall here the Riemann-Roch formula for a curve, is the case of Riemann surfaces or Riemann surfaces with marked points which interests us here: $\mathcal{M}_{g}, \mathcal{M}_{g, n}$ respectively. That need to define The moduli space of curves of genus $g$, and the moduli space of curves of genus $g$ with $n$ marked points:

\section{Riemann Roch formula for a curve}

The Riemann-Roch formula gives:

$$
\operatorname{dim}_{\mathbb{C}} H^{0}(T \Sigma)-\operatorname{dim}_{\mathbb{C}} H^{1}(T \Sigma)=\int_{\Sigma} \operatorname{ch}(T \Sigma) t d(T \Sigma)
$$

Specifically, $\operatorname{dim}_{\mathbb{C}} H^{0}(T \Sigma)$, consider the infinitesimal automorphisms, $\operatorname{dim}_{\mathbb{C}} H^{1}(T \Sigma)$ is the complex dimension of moduli space of curves. For a Riemann surface of genus $g$ yields: Dimension of $\mathcal{M g}$ :

$$
\begin{gathered}
\operatorname{dim}_{\mathbb{C}} H^{0}(T \Sigma)-\operatorname{dim}_{\mathbb{C}} H^{1}(T \Sigma)=3-3 g \\
\operatorname{dim}_{\mathbb{C}} \mathcal{M}_{g}=3 g-3+\operatorname{dim}_{\mathbb{C}} H^{0}(T \Sigma)
\end{gathered}
$$

Riemann-Roch formula for a map, curves, stable maps Make first application of the formula above: $H^{0}(T \Sigma)$ count the number of marked points need to stabilize the curve thus:

$\left.g \geq 2: \operatorname{dim}\left(H^{0}(T \Sigma)\right)=0\right): \operatorname{dim}\left(H^{1}(T \Sigma)\right)=$ $\operatorname{dim}\left(\mathcal{M}_{g}\right)=3 g-3$

if $g=1: H^{0}(T \Sigma)=\mathbf{C}: \operatorname{dim}\left(H^{1}(T \Sigma)\right)=$ $\operatorname{dim}\left(\mathcal{M}_{g}\right)=1$

if $g=0: \operatorname{dim}\left(H^{0}(T \Sigma)\right)=3: H^{1}(T \Sigma)=\mathcal{M}_{g}$ is a point.

We will therefore focus our attention on stable curves (and maps). Let $\phi: \Sigma \rightarrow X$ be a holomorphic curve The Riemann-Roch formula gives in this case:

$$
\begin{array}{r}
\operatorname{dim}_{\mathbb{C}} H^{0}\left(\phi^{*} T X\right)-\operatorname{dim}_{\mathbb{C}} H^{1}\left(\phi^{*} T X\right) \\
=\int_{\Sigma} \operatorname{ch}\left(\phi^{*} T X\right) t d(\Sigma)=n(1-g)+\int_{\Sigma} \phi^{*} c_{1}(T X)
\end{array}
$$

\subsection{Curves, stable maps, moduli spaces}

In genus 0 , an automorphism of $P^{1}(\mathbb{C})$ is determined by the images of three distinct points, that is to say automorphisms which fix two points form a no discrete subgroup $P G L(2, \mathbb{C})$,

we will therefore infinitesimal automorphisms. To get rid of infinitesimal automorphisms: stabilize the curve, we must add "marked points" doing the curve is stabilized.

A stable curve is a curve on which we added enough marked points to kill the infinitesimal automorphisms. A stable map is a parametric curve $\left(\Sigma,\left(x_{1}, \ldots, x_{k}\right), \varphi\right)$ for which $\Sigma$ is stable. We can now give the definition of the moduli space of stable maps with $k$ marked points: $\mathcal{M}_{g, n}(X, \beta)$.

We define the space:

$\mathcal{M}_{g, n}(X, \beta)=\left\{\left(\Sigma,\left(x_{1}, \ldots, x_{n}\right), \varphi\right), \varphi: \Sigma \rightarrow\right.$ $\left.X, \varphi_{*}(\Sigma)=\beta\right\} / \sim$ where $\sim$ are quotient by the group of reparameterization.

The space considered above is not compact in general, we denote $\overline{\mathcal{M}}_{g, n}(X, \beta)$ its compactification, this amounts to add singular curves .

\section{6 (Virtual) dimension space of holomor- phic curves}

From the two previous formula (Riemann-Roch for curve and parametric curve), we can deduce the "virtual" dimension of moduli space of holomorphic curves. For this we can use an exact sequences. Consider the long exact sequence in cohomology associated to the exact sequence:

$0 \rightarrow T_{\Sigma} \rightarrow f^{*} T_{X} \rightarrow N_{\Sigma / X} \rightarrow 0$

For details see Pandharipande works [9]

The Dimension of $\mathcal{M}_{g}(X)$ is given by combining the two forms of the Riemann-Roch previous:

$\operatorname{dim}_{\text {virt }} \overline{\mathcal{M}}_{g, n}(X, \beta)=(\operatorname{dim} X)(1-g)+$ $\int_{f_{*}(\Sigma)} c_{1}(T X)+3 g-3+n$

We could find directly this result in symplectic case (varieties that are treated are thus symplectic Kahler) relying on the index of a Fredholm operator for an elliptic complex adhoc.

The spaces of modules of curves are compacted to take account of singularities, and stabilized by quotient by a group of automorphism. They correspond to orbifolds for physicists . In the language of algebraic geometry, the adapted structure is that of algebraic stack. We can determine a (virtual) dimension to these spaces given by Riemann Roch's theorem which is a rigid version of the Atiyah Singer index theorem. One application in mathematics is the counting of curves passing through a certain number of marked points. it is enumerative geometry. In Mathematical Physics, the correlation functions resulting from this 
theory leads to the invariants of Gromov Witten (Theory of supersymmetric strings, A- Model of Witten), or the invariants of Donaldson [10] For the study of four manifolds.

\section{Gromov Witten invariant, A-Side of mirror symmetry}

Witten [11], to show that the introduction of supersymmetry, made it possible to localize incalculable path integrals around the critical configurations.

that are instantons. In the case of the superstring theory, the A-model shows that these instantons are precisely the holomorphic curves satisfying the Cauchy Riemann conditions. Suddenly the path integrals become computable in particular, the correlation functions can be seen as the invariants of Gromov Witten. In the appropriate cases, those where the instabilities have been killed, these invariants become numbers. is called Gromov-Witten invariant quantity:

$<\left[\omega_{1}\right], \ldots\left[\omega_{n}\right]>_{\beta}=\int_{\left[\overline{\mathcal{M}}_{g, n}(X, \beta)\right]} e v^{*}\left(\left[\omega_{1}\right]\right) \wedge \ldots \wedge e v^{*}\left(\left[\omega_{n}\right]\right)$

In this script we used an evaluation map:

$$
e v_{i}: \overline{\mathcal{M}}_{g, n}(X, \beta) \rightarrow X:\left(\Sigma, x_{1}, \ldots, x_{n}, \varphi\right) \mapsto \varphi\left(x_{i}\right)
$$

and the dual map:

$$
e v_{i}^{*}: H^{*}(X) \rightarrow H^{*}\left(\overline{\mathcal{M}}_{g, n}(X, \beta)\right):\left[\omega_{i}\right] \mapsto e v_{i}^{*}\left(\left[\omega_{i}\right]\right)
$$

Kontsevich [12], early nineteen nineties gave an elementary model by considering the holomorphic curves of $P^{1}(\mathbb{C})$ in $P^{2}(\mathbb{C})$, he thus obtained an iterative formula allowing to know by how many point passes a curve of any degree: Inspired by Feymann diagram he showed that There $N_{d}$ the number of curves of degree $d$ through $3 d-1$ points, given by:

$$
N_{d}=\sum_{d=d_{1}+d_{2}} N_{d_{1}} N_{d_{2}}\left(d_{1}^{2} d_{2}^{2} C_{3 d_{1}-2}^{3 d-4}-d_{1}^{3} d_{2} C_{3 d_{1}-1}^{3 d-4}\right)
$$

By applying this formula, we find: $N_{2}=1, N_{3}=12$, $N_{4}=620$

\subsection{Back physics!}

We return now to our case for the supersymmetric $A$ model of Witten, take $X$ Calabi-Yau 3 included in $\mathbb{P}^{4}(\mathbb{C})$

It is known that Calabi Yau Ricci-flat is that means:
$\int_{\Sigma} \varphi^{*}\left(c_{1}(X)\right)=0$ In These conditions, the dimension of $\overline{\mathcal{M}}_{g, n}(X, \beta)$ is given by:

$$
\operatorname{dim}\left(\overline{\mathcal{M}}_{g, n}(X, \beta)\right)=-3+3 g+\operatorname{dim} X(1-g)+n
$$

If $g=0$ and $\operatorname{dim} X=3$ we get: $\operatorname{dim}\left(\overline{\mathcal{M}}_{0, n}(X, \beta)\right)=$ $n$

In $\left.\overline{\mathcal{M}}_{0, n}(X, \beta)\right)$ as $\operatorname{deg}([H])=\operatorname{codim}[H]=1$ it takes $n$ hyperplanes in $<[H], \ldots,[H]>_{\beta}$ to make this non-trivial correlation function.

For $n=3$, we have the three points correlation function or "pant": $n=3,<[H],[H],[H]>_{\beta}$, basic "lego" of topological fields theory .

\subsection{Example 2: Calculating the correlation function at three points: the problems, the mirror symmetry}

The calculation of correlation functions by direct methods namely 'A'side of the mirror is not easy: if you expand you have:

$<[H],[H],[H]>=\sum_{\beta}<[H],[H],[H]>_{\beta}$ $e^{-\int_{\beta} \omega}$.

A conjecture of Clemens [13] said we cannot calculate this number due to a problem of multiple coverings, which contradicts the expected dimension of 0 for space applications module with 0 runs scored.

To survive we must work on the other side of the mirror. mirror symmetry The objects we work with in enumerative geometry are Kahler forms and parameterize deformations of this form. Mirror symmetry says that one can express the same physical setting deformations of complex structure on a variety mirror and identifying the correlation functions from the two models.

\section{Mirror symetry for closed strings}

The mirror symmetry postulate that there are two viewpoints to represent the physics of fermionic strings. The relevance comes from the fact that some problems not solvable from a certain point of view are on the other. Mirror symmetry is a very accomplished form of T-duality: appeared in bosonic theory which states that the partition function remains unchanged in the change $R \leftrightarrow \frac{1}{R}$, where $R$ denotes the radius of compactification of extra dimension. In a bosonic field theory, where the source space is the cylinder and target a torus, one can notice that the T-duality exchange symplectic structure deformation (area) and deformation of complex structure. In this "survey", we will introduce tools from physics and mathematics for understanding some aspects of mirror symme- 
try. Especially we construct the mirror map locally as historically Morrison [14] has presented this theory for closed strings. In addition, we briefly explain the implications in enumerative geometry.

\subsection{Theory $\varphi^{4}$ and complex geometry}

The model of Ginzburg Landau plays a decisive role on the B-side of the mirror. We will look at the admissible deformations ( marginal distortions ) preserving certain symmetries. Starting from the Lagrangian in $\varphi^{4}$ :

$\mathcal{L}_{L G}=\partial_{\mu} \phi \partial_{\mu} \phi-V(T, \phi)$ where $V(T, \phi)=$ $\frac{1}{4 !} \lambda(t) \phi^{4}+\frac{1}{2 !} \mu^{2}(t) \phi^{2}$ At the critical temperature $T_{c}$ "mass", $\mu^{2}\left(T_{c}\right)=0$, so the correlation length (inverse mass) is infinite. At this temperature the field $\phi_{0}$, solution of $\frac{\partial}{\partial \phi} V(T, \phi)$ is zero three times degenerated. A small perturbation $V\left(T_{c}, \phi\right) \rightarrow V\left(T_{c}, \phi\right)+$ $\delta \mu^{2}(T) \phi^{2}$, solves the singularity and the is "symmetry breaking". The challenge is to find ways to perturb marginally a potential in order to preserve the symmetry and defined by the fact a critical family of superpotentials.

\subsection{Marginal deformations, Calabi-Yau hy- persurfaces}

The superpotential is an holomorphic function $\mathcal{W}: \mathbb{C}^{M} \rightarrow \mathbb{C}$ is chosen as a potential $V(x)=\sum_{1}^{N}\left|\partial_{i} \mathcal{W}(X)\right|^{2}=\sum_{1}^{N} \partial_{i} \mathcal{W}(X) \partial_{i} \mathcal{W}(X)^{*}$ if we consider only one field, one can consider the function $\mathcal{W}(X)=\frac{1}{(n+1) !} X^{n+1}$

The bosonic part of the supersymmetric Lagrangian is written then:

$\mathcal{L}_{N=2}^{L G}=-\partial_{+} X^{*} \partial_{-} X+\partial_{-} X^{*} \partial_{+} X+V(X)$

There is: $V(X)=0 \Leftrightarrow \partial_{i} \mathcal{W}\left(X_{0}\right)=0$, so it is relevant to define the Chiral ring $\mathcal{R}_{\mathcal{W}}=\frac{\mathbb{C}[X]}{\partial \mathcal{W}(X)}$ where the ratio is proportional to the polynomials of $\partial_{i} \mathcal{W}(X): P(X)=P^{i}(X) \partial_{i} \mathcal{W}(X)$ deformations respecting the Chiral ring are given by: $\mathcal{W}_{\text {def }}(X)=\mathcal{W}(X)+\sum_{P \in \mathcal{R}_{\mathcal{W}}} t_{P} P(X)$

If we choose $\mathcal{W}(X, Y, Z)=\frac{1}{3}\left(X^{3}+Y^{3}+Z^{3}\right)$, the deformed potential is given by $\mathcal{W}_{\text {def }}(X, Y, Z)=$ $\mathcal{W}(X, Y, Z)+t_{0}+t_{1} X+t_{2} Y+t_{3} Z+t_{4} X Y+$ $t_{5} Y Z+t_{6} Z X+t_{7} X Y Z$

Only the non vanishing term $\mu=t_{7}$ preserves the critical situation, and does not break the $\mathbb{Z}_{3}$ symmetry $(X, Y, Z) \rightarrow\left(\exp \left(\frac{2 k i \pi}{3}\right) X, \exp \left(\frac{2 k i \pi}{3}\right) Y, \exp \left(\frac{2 k i \pi}{3}\right) Z\right)$ we have defined a family of continuous deformations allowed:

$\mathcal{W}_{\text {def }}(X, Y, Z, \mu)=\frac{1}{3}\left(X^{3}+Y^{3}+Z^{3}\right)+\mu X Y Z$ of the initial potential. The hypersurface of a complex projective space obtained by canceling this is the The simplest example of Calabi-Yau. Is an elliptic curve or complex torus .Calabi-Yau variety is a Kahlerienne Ricci flat which is to say: the canonical bundle is trivial. There are, among other, $K 3$ surfaces involved in branes theory and the quintic threefold for closed strings. The easiest way to realize the Calabi-Yau is to consider an hypersurface of a complex projective space. There is a strong constraint between the degree of a hypersurface and the dimension of the ambient space. Subsection Example of a Calabi-Yau Let us write the exact sequence associated to a hypersurface of degree $d$ :

$0 \rightarrow \mathcal{O}_{\mathbb{P}^{n+1}}(-d) \rightarrow \mathcal{O}_{\mathbb{P}^{n+1}} \rightarrow \mathcal{O}_{X} \rightarrow 0$

With exact long sequence in cohomology, one can calculate the cohomology groups associated.

The Result: $H^{n}\left(X, \mathcal{O}_{X}\right)=\mathbb{C}^{C_{n-1}^{d-1}}=\mathbb{C}$ (condition Calabi-Yau) Necessarily $d=\mathrm{n}+2$

$$
\begin{aligned}
& \text { - } n=1(\mathrm{~d}=3) \text { : } \text { Elliptic Curves } \\
& \text { - } n=2(\mathrm{~d}=4) \text { : K3 Surfaces } \\
& \text { - } n=3(\mathrm{~d}=5) \text { : Quintic threefold }
\end{aligned}
$$

\subsection{Deformations, mirror symmetry}

We can focus on two types of deformations of CalabiYau: the deformations of symplectic structure and those from its complex structure. The tangent space of complex structure is $H^{1}(X, T X) \simeq H^{2,1}(X)$, can consider the deformation of complex structure ( $\boldsymbol{J}$ deformation). the Kähler structure is governed by $H^{1,1}(X)=H^{1}\left(X, \Omega_{X}^{1}\right)$ There are two field theories ( textbf CFT) supersymmetric duality in satisfactory: $h^{2,1}(X)=h^{1,1}(M X)$ and $h^{1,1}(X)=h^{2,1}(M X)$ : deform the complex structure of $X$ is equivalent to deform the volume of his mirror.

diamond Hodge plots the Hodge numbers of a complex manifold

Hodge Diamond for Calabi-Yau Quintic Threefold

$$
\begin{array}{cccccc} 
& & h^{0,0}=1 & & & \\
h^{3,0}=1 & h^{2,0}=0 & h^{1,0}=0 & h^{0,1}=0 & & \\
& h^{2,0}=0 & \frac{h^{2,1}=101}{h^{1,1}=1} & h^{1,2}=101 & h^{0,2}=0 & h^{0,3}=1 \\
& h^{1,0}=0 & h^{1,1}=1 & h^{0,1}=0 & h^{0,2}=0 & \\
& & h^{0,0}=1 & & &
\end{array}
$$

\section{4 "B side" Origin of Physics}

On the $A$ Side, supersymmetric constraints lead to what the action does depends only on the the Kähler form; instantons are holomorphic curves. The calculation of correlation functions is difficult because it 
takes into account correction on the degree curves (invariants of Gromov-Witten) On the side "B", BRST Formalism explained in the other side of the mirror applies here: instantons are constants maps from the world-sheet $\Sigma$ on a point of target space $X$. The correlation functions are simpler to calculate: they require no instanton correction.

If $X$ is a Calabi-Yau 3, the 3 points correlation function is:

$\left\langle W_{A} W_{B} W_{C}\right\rangle=\int_{X} \Omega^{j k l} A_{j} \wedge B_{k} \wedge C_{l} \wedge \Omega$

$A, B, C$ belong to $H^{1}(X, T X)$ and depend on the complex structure, $\Omega$ is (3.0) top-form holomorphic. The two numbers $h^{1,1}(X)=1$ and $h^{2,1}(X)=101$, count the number of deformation structures respectively Kähler and complex. The principle of mirror symmetry, gives $h^{1,1}(M X)=101$ and $h^{2,1}(M X)=1$. He said in addition that correlation functions calculated from both sides of the mirror are identical . The Mirror map associated parameter of deformation of Kalher structure with parameter of deformation of complex structure. If a problem is difficult at the $A$ side, we can try to solve it at the $B$ side. In mathematics passing through the mirror application can solves so important old problem of Enumerative geometry

\subsection{The Quintic and its mirror}

Recall that the homogeneous quintic in $\mathbb{P}^{4}$, is obtained by canceling the superpotential: $\mathcal{W}=$ $\frac{1}{5}\left(X_{0}^{5}+\ldots+X_{4}^{5}\right)$

A marginal deformation of this superpotential is almost the variety expected the variety mirror quintic is associated with the crepant resolution of:

$$
\begin{aligned}
& \left\{\left(X_{0}, \ldots, X_{4}\right) \in \mathbb{P}^{4} / \frac{1}{5}\left(X_{0}^{5}+\ldots+X_{4}^{5}\right)-\mu X_{0} \ldots X_{4}=\right. \\
& 0\}_{/ G} \\
& \text { with } G=\left\{\left(a_{0}, \ldots, a_{4}\right) \in \mathbb{Z}_{/ 5} / \sum a_{i}=\right. \\
& 0\}_{/ \mathbb{Z} / 5=\{(a, a, a, a, a)\}} \simeq\left(\mathbb{Z}_{/ 5}\right)^{3}
\end{aligned}
$$

\subsection{Program}

location The overall construction of the mirror map is difficult. Should be localize and built the map at the neighborhood of a point.

Plan of the study

$X$ denote the Calabi-Yau, $M X$ its mirror .

- We must first calculate the Yukawa coupling $H^{1}(M X, T M X)$
- Identify by the mirror map.

- Deduce the Yukawa couplings of $H^{1,1}(X)$

One can deduce predictions about the number of rational curves in $X$ We will briefly describe in the following useful mathematics for the $B$-side the Quintic.

\subsection{Mirror-map}

The principle of mirror symmetry says that $<H, H, H>=<\theta, \theta, \theta>$, if $t H$ denotes an curve parameter in the module of Kahler $X$ we set $H=\frac{d}{d t}=2 \pi i q \frac{d}{d q}$ its tangent vector $(q=\exp (2 \pi i t)$ Local coordinates for this module.

The problem is to produce an image $q(x)$ in module complex deformations was: $q=q(x), \frac{d}{d q} \rightarrow \frac{d x}{d q} \frac{d}{d x}$ Then we can write $\left.H=2 \pi i q \frac{d}{d q}\right) \leftrightarrow \theta=2 \pi i q \frac{d x}{d q} \frac{d}{d x}$ $<H, H, H>=\left(2 \pi i q \frac{d}{d q}\right)^{3}<\frac{d}{d x}, \frac{d}{d x}, \frac{d}{d x}>=$ $\left(2 \pi i \frac{q}{x} \frac{d x}{d q}\right)^{3}<x \frac{d}{d x}, x \frac{d}{d x}, x \frac{d}{d x}>$

\subsection{Mathematical Tools}

The simplest examples of Calabi-Yau are elliptic curves. They will guide us to understand the techniques identified below and set the mirror application. Monodromy: For a flat bundle,go around a singular object centred in $t=0$ at constant distance $|t|,(t$ :deformation parameter of a smooth family) is namely monodromy in mathematics. In physics we talk about loop Wilson. Residue map: We can generalize the formula for residues of a complex variables function around $z=0$ by replacing function differential forms and point by hypersurface. This will be very useful for calculating periods.

\subsection{Elliptic curves}

The elliptic curve $E_{\tau}=\mathbb{C} /(1, \tau)$ is a Calabi-Yau of complex dimension 1 . The volume form is given by $\Omega=d z$

Hodge Numbers: Hodge diamond is:

$$
\begin{array}{lllll} 
& h_{00} & & 1 & \\
h_{10} & & h_{01}=1 & & 1
\end{array}
$$

The one parameter family of deformations of an elliptic curve is:

$X^{3}+Y^{3}+Z^{3}-3 \psi X Y Z=0$

If $\alpha$ and $\beta$ are homology cycles, they depend then $\psi$, we can find $\tau$ to from ratio periods: $\int_{\alpha} \Omega, \int_{\beta} \Omega$. Solving a differential equation called Picard-Fuchs 


\subsection{Family of elliptic curves, monodromy}

\section{degeneration of a family of curves}

$\mathrm{X}[\mathrm{d}]_{\pi} \supset X_{t}[d] t \neq t^{\prime} X_{t} \simeq X_{t}^{\prime}$

$D^{2} \ni t t=0 X_{0}$ : sing.

Theoremmonodromy : Either $\mathrm{X}_{t}$ where $t$ varies along a loop in $\pi_{1}\left(D^{2}-\{0\}, t_{0}\right)$ around 0 , (Wilson loop), all elements of this family are diffeomorphic .This induces an automorphism on homology: $\left.\varphi_{\star} \in \mathcal{A} u t\left(H_{n}\left(X_{t_{0}}\right), \mathbb{Z}\right)\right)$ Pratical Example Let's illustrate by taking a one parameter family of elliptic curve $C_{t}=\left\{\left(Y^{2} Z=X^{3}+X^{2} Z-t Z^{3}\right\} \subset \mathbb{C P}^{2}\right.$ which is expressed

in affine coordinates: $C_{t}: y^{2}=x^{3}+x^{2}-t$ : elliptic curve defined by an algebraic equation.

The parameter $t$ of the elliptic curve is the signature of the variation of complex structure, the geometric expression is $E_{\tau}=\mathbb{C} /(1, \tau(t))$ when $t$ revolves around the origin $\tau(t) \rightarrow \tau(t)+1$ with $\tau$ function of: $t: \tau(t)=\frac{\ln t}{2 \pi i}$

At the automorphism group:

$\left(\begin{array}{c}\tau(t) \\ 1\end{array}\right) \rightarrow\left(\begin{array}{ll}1 & 1 \\ 0 & 1\end{array}\right)\left(\begin{array}{c}\tau(t) \\ 1\end{array}\right)=\left(\begin{array}{c}\tau(t)+1 \\ 1\end{array}\right)$

We find that the complex structure varies but the symplectic structure is unchanged.

We justify the choice of new coordinate $q(t)=$ $e^{2 \pi i \tau(t)}$, because a passage to the limit gives: when $t \rightarrow 0, \operatorname{Im} \tau(t) \rightarrow+\infty$, so $q(t)$, where $q(t)$ is an holomorphic function of $t$ which also go to 0 ; so it is a local coordinate for this family of elliptic curves. We spoke in physics: large complex structure limit(WSCC) in "bijection" with large volume limit ( $\boldsymbol{L V} \boldsymbol{L}$ ) on the $A$ side

\subsection{Application to the quintic}

Is the quintic $\sum_{i=0}^{4} x_{i}^{5}-\psi x_{0} x_{1} x_{2} x_{3} x_{4}=0$. As with elliptic curves, Morrison showed by standing near $x=\left(\frac{1}{\psi}\right)^{-5}=0$ we could find $t$ (side strain Kähler ) from of $\psi$ (or $x$ ).

As with elliptic curves, $t=\frac{\int_{\gamma_{1}} \Omega}{\int_{\gamma_{0}} \Omega}$, we must calculate the periods $\phi_{i}(x)=\int_{\gamma_{i}} \Omega, i=1,2 . \gamma_{0}$ is invariant under monodromy around $x=0$ (large complex structure limit (WSCC )), $\psi \rightarrow \infty \psi \rightarrow \infty \gamma_{1} \rightarrow \gamma_{0}+\gamma_{1}$. Both quantities depend on $\psi$ (or $x$ )

We can compute locally the three forms (*) $\Omega$ using a version "differential form" of theorem of residue (and the theorem of implicitfunction) and deduce by direct calculation, the first period.

The equation called Picard Fuchs calculates the other period. Finally, we find:

$\phi_{0}(x)=\sum_{n=0}^{\infty} \frac{5 n !}{(n !)^{5}} x^{n}$

$\phi_{1}(x)=\phi_{0}(x) \log (x)+f(x)$,

with

$f(x)=5 \sum_{n=0}^{\infty} \frac{5 n !}{(n !)^{5}}\left(\sum_{j=n+1}^{5 n} \frac{1}{j}\right) x^{n}$

\subsection{Calculation of Yukawa couplings quoted $B$}

Let $\Theta^{(i)}=\left(x \frac{d}{d x}\right)^{(i)}$, the equation of Picard-Fuch written:

$\Theta^{(4)} y+\frac{2.5^{5} x}{1+5^{5} x} \Theta^{(3)} y+\frac{7.5^{4} x}{1+5^{5} x} \Theta^{(2)} y+\frac{2.5^{4} x}{1+5^{5} x} \Theta^{(1)} y+$ $\frac{2.5^{5} x}{1+5^{5} x} \Theta y=0$

It is applied to $\Omega$ :

If $Y=\int_{M X} \Omega \wedge \Omega^{(3)}, \int_{M X} \Omega \wedge \Omega=\int_{M X} \Omega \wedge \Omega^{\prime}=$ $\int_{M X} \Omega \wedge \Omega "=0$, twice differentiating the last equality we have: $\int_{M X} \Omega \wedge \Omega^{(4)}+2 \int_{M X} \Omega^{\prime} \wedge \Omega^{(3)}=0$

We deduce the differential equation $\left(x \frac{d}{d x}\right) Y=$ $\frac{-5^{5} x}{1+5^{5} x} Y$

The solution is $Y=<x \frac{d}{d x}, x \frac{d}{d x}, x \frac{d}{d x}>=\frac{c_{2}}{1+5^{5} x}$

We must normalize $Y$ in agreement with $(*)$ and then divide $\Omega$ by $\Phi_{0}(x)$ Thus:

$<x \frac{d}{d x}, x \frac{d}{d x}, x \frac{d}{d x}>=\frac{c_{2}}{\left(1+5^{5} x\right) \phi_{0}(x)^{2}}$ For identification,

$<H, H, H>=\left(2 \pi i \frac{q}{x} \frac{d x}{d q}\right)^{3}<x \frac{d}{d x}, x \frac{d}{d x}, x \frac{d}{d x}>=$ $\frac{c_{2}\left(2 \pi i \frac{q}{x} \frac{d x}{d q}\right)^{3}}{\left(1+5^{5} x\right) \phi_{0}(x)^{2}}$

\subsection{Conclusions: Application to enumera- tive geometry}

The parameter $t$ Kahler deformation, expressed as a function of the ratio of the first two periods, we get: $q=e^{2 i \pi \frac{\phi_{1}(x)}{\phi_{0}(x)}}$ Where:

$q=c_{1}\left(x-770 x^{2}+\ldots\right)$ and conversely $x=\frac{q}{c_{1}}+770\left(\frac{q}{c_{1}}\right)^{2}+\ldots$

We can now calculate everything according to the variable $q$ :

$<H, H, H \quad>=\quad(2 \pi i)^{3}\left(-c_{2}-575\left(\frac{c_{2}}{c_{1}}\right) q-\right.$
$\left.19575\left(\frac{c_{2}}{c_{1}^{2}}\right) q^{2}+\ldots\right)$ It remains to calculate the constants $c_{1}$ and $c_{2}$.

We remember that it was not enumerate rational curves of degree $n_{d}$ is known for first degrees, which calculates $c_{1}$ and $c_{2}$.

$<H, H, H>=5+\sum_{d=1}^{\infty} n_{d} d^{3} \frac{q^{d}}{1-q^{d}}=5+2875 q+\ldots$ 
We deduce $c_{2}=\frac{-5}{(2 \pi i)^{3}}, c_{1}=-1$ Finally, we can enumerate the number curve of a rational quintic of $\mathbb{P}^{4}$ all degrees $<H, H, H>=5+\sum_{d=1}^{\infty} n_{d} \frac{d^{3} q^{d}}{1-q^{d}}=$ $\mathbf{5}+\mathbf{2 8 7 5} \frac{q}{1-q}+\mathbf{6 0 9 2 5 0} .2^{3} \frac{q^{2}}{1-q^{2}}+\ldots$

\section{Homological mirror symmetry}

It was previously presented a mirror symmetry involving the closed strings, supposed to translate gravitation. One may wonder if such a theory can be adapted to open strings: the answer is yes. We have seen that mirror symmetry was an elaborate form of $T$-duality. But how to translate the number of winding in the case of open strings. The answer to the previous question exists: you can wind an open string provided that its ends are fixed on a branes. Thus the mirror symmetry of the open strings is based on this duality "open strings-branes".

In axiomatic theory of homology, we often consider the relative homology of a space by a subspace: $H_{*}(A, B)$. In fact we can do the same in topological field theory; One can replace in theory of fields the source space $\Sigma$ by the pair $(\Sigma, \partial \Sigma)$, and thus think, for example of the evolution of an open string whose extremities are constrained to remain fixed on a brane(s). This approach would describe all of the particles, not just gravitation. in this theory, in fact, the particles of the standard model $U(1) \times S U(2) \times S U(3)$ are seen as open strings fixed on a brane: the universe in which we evolve. A $D$ - Geometric Brane is a triple $\left(L, E, \nabla_{E}\right)$ where $L$ is a sub-manifold of dimension real p: $L$ included in $(M, E)$, a vector bundle and $\nabla_{E}$ a connection on $E$. An open string of origin $\left(L, E, \nabla_{E}\right)$ and target $\left(K, F, \nabla_{F}\right)$ is a map $X$ from $I=[0,1] \in M$ with $X(0) \in L, X(1) \in K$. We also call "open string story", a map from of the open Riemann surface. $\Sigma=I \times \mathbb{R} \in M$ with $X(0, t) \in L$ and $X(1, t) \in K$ these fields are supposed to be holomorphic curves on the $A$ side: Symplectic side. But why a bundle $E$ and a connection?: A elementary particle is charged by a gauge field for the electron it is the Maxwell field of group $U(1)$, For a quark the charge is its color: Yang Mills field, so we associate with an elementary particle a bundle $E$ and its associated connection. By analogy, at each end of an open string, is associated the couple $\left(E, \nabla_{E}\right)$. In the story of a particle, or of the ends of an open string, we are led to consider the holonomy of the connection (linear map) from $\left.E\right|_{\phi\left(t_{1}\right)}$ to $\left.E\right|_{\phi\left(t_{2}\right)}$. By identifying the $N$ branes between them, we can describe the interactions be- tween open strings. At the origin of an open string living on the brane $i$, we specify the target brane $k$. we obtain an index called Chan-Paton . the introduction of $N$ branes therefore introduces a gauge theory $U(N)$. This theory is non commutative if $n>1$. One brane is necessary to describe the electomagnetism, at least 2 are necessary to describe interactions involving the gauge group $S U(2)$. By playing with this, we can model the different theories of gauges. To find particles in this modeling, it is necessary to consider intersections of branes: this is the case where the length of the rope joining two branes tends towards 0

\subsection{Categorization of mirror symmetry and open string theory}

To define the mirror symmetry of open strings, Kontsevich categorizes the complex-symplectic exchange. He invents homological mirror symmetry.The main idea is to replace the objects of mirror symmetry, namely the symplectic manifolds by objects belonging to two categories "mirrors of each other": namely chain complexes. The Fukaya category contains the chain complexes on the symplectic side (side $A$ of the mirror), a subcategory of coherent sheaves, modelize the complex geometry on side $B$. The mirror application will also be replaced by a functor between these the two categories. Is not the first example of field theory to use category language: Chern-Simon theory uses categorifications from knot-theory. Kontsevich's statement in 1994 says that:

There is an equivalence of category between The bounded derived category of coherent sheaves $D^{b}(X)$ on Calabi- Yau $X$ and Fukaya category of its mirror $M X$.

\subsection{Symplectic geometry side A}

Briefly, We will always consider in this context, holomorphic curves but now the Riemann's surfaces will be a surface with boundary We will describe the curves in this context more precisely. A holomorphic curve (associated with lagrangians $L_{1}, L_{2}$ ) in a symplectic variety $(M, \omega)$ is:

$u: \mathbb{R} \times[0,1] \rightarrow M$ with $J$ an almost complex structure such as:

$\bar{\partial}_{J} u=0$ : Cauchy Riemann conditions, $u(s, 0) \in L_{0}$, $u(s, 1) \in L_{1}$

$\lim _{s \rightarrow+\infty} u(s, t)=p, \lim _{s \rightarrow-\infty} u(s, t)=q$

We can stay on the $A$ side and do enumerative geometry: Curve counting (holomorphic discs). 
Here the option will be to categorize from Floer's homology and to find an equivalent category in the $B$ side. On the symplectic side, in open string theory the good theory is Floer's homology. This homology is a kind of of Morse homology adapted to the language of symplectic geometry. It was invented by Floer to demonstrate Arnold's conjecture in symplectic geometry.Formally the Floertheory is an adaptation of that of Morse in infinite dimension. The starting manifold is replaced by a path space. Instead of critical points, we will have to define the notion of "critical paths ..."

Let $\mathcal{P}\left(L_{0}, L_{1}\right)$ be the space of the end paths in the two Lagrangian $L_{0}$ and $L_{1}$; We then define a functional of the space of (homotopies) of paths $\gamma$ in $\mathbb{R}$ :

$(\gamma,[u]) \rightarrow \int_{[0,1]^{2}} u^{*} \omega$

this quantity represents the symplectic area which replaces the height function of Morse theory.

Let $v$ a vector field, The differential of the previous expression is $d \mathcal{A} \cdot v=\int_{[0,1]} \omega(\dot{\gamma}, v) d t$ : critical points are constant paths (immediate).

The trajectories of the gradient are

\section{J-holomorphic maps}

\subsubsection{Floer's Complex}

Let: $C F\left(L_{0}, L_{1}\right)=\Lambda^{\left|L_{0} \cap L_{1}\right|}$, free module generated by the points of intersection of the two Lagrangian $L_{0}$ and $L_{1}$, we define a differential:

$\partial p=\sum_{\operatorname{deg}(q)=\operatorname{deg}(p)-1}(\mathcal{M}(p, q,[u], J) / \mathbb{R}) T^{\int u * \omega} q$ As in Morse homology, we show that $\partial$ defines a complex:

broken trajectories $\coprod_{r \in L_{0} \cap L_{1}}(\mathcal{M}(p, r,[u], J) / \mathbb{R}) \times$ $(\mathcal{M}(r, q,[u], J) / \mathbb{R})$

compacts a space of dimension 1 so add a even number of points at the edges: the square of the edge is therefore zero

Note: in the exact Lagrangian case, we can avoid $T^{\int u^{*} \omega}$ and work modulo 2, exactly as in Morse, We just defined the Floer complex! The physicist will be able to see that there is an homology managing the brane intersection points ...

\subsubsection{Categorization of Floer's homology}

We can define a product structure on the Floer complexes:

$C F^{*}\left(L_{0}, L_{1}\right) \otimes C F^{*}\left(L_{1}, L_{2}\right) \rightarrow C F^{*}\left(L_{0}, L_{2}\right)$

The module space will be $\mathcal{M}(p, q, r,[u], J))$ of dimension $\operatorname{deg}(r)-(\operatorname{deg}(p)+\operatorname{deg}(r)$

The product: $p . q=\sum_{r, \operatorname{deg}(r)=\operatorname{deg}(p)+\operatorname{deg}(q)}(\mathcal{M}(p, q, r,[u], J) / \mathbb{R}) T^{\int u^{*} \omega} r$ we can reiterate:

$C F^{*}\left(L_{0}, L_{1}\right) \otimes C F^{*}\left(L_{1}, L_{2}\right) \ldots \otimes C F^{*}\left(L_{n-1}, L_{n}\right) \rightarrow$ $C F^{*}\left(L_{0}, L_{n}\right)$ We can then define a $\infty$-category whose objects are Lagrangian, and morphisms are the Floer complexes, . We categorized the symplectic side..

\section{Complex geometry on B-side}

On this side we have already seen that dominates complex geometry. his categorization will lead to define the category of coherent sheaves. We cannot give more details here. As in the case of closed strings, it will be a question of defining an mirror map allowing to go from side B to side A. The first example that validates this theory was given by Zaslow [15], in the context of the Lagrangian associated with elliptic curves (complex torus).

\section{Conclusion}

Unfortunately, in this paper we cannot mention all the studies undertaken so far. It seems that the noncommutativity that we discover in boundary field theories is interesting. it evokes the symmetry groups of the particles of the standard model. Another approach proposed by Alain Connes is possible, it is non-commutative geometry. The theory of the index can also be considered in this context of noncommutativity. In his theory, Alain Connes does not need to speak about supersymmetry. It uses a triplet $(\mathcal{A}, \mathcal{H}, \mathrm{D}), \mathcal{A}$ being the deformation of the algebra of continuous functions :semi-direct product of algebra of $\mathrm{C} \infty$ functions on a manifold by matrice spaces accounting for the internal symmetries of the particles of the standard model. It might be interesting to understand the links between the elaborate gauge theories proposed by Witten using supersymmetry and that of Alain Connes using non-commutative algebras ...

\section{References:}

[1] D.McDuff, D.Salamon, J-holomorphic Curves and Quantum Cohomology, Univ. Lecture series 6, A.M.S., 1994.

[2] M. F. Atiyah and I. M. Singer The index of elliptic Operators on compact manifolds, Journal: Bull. Amer. Math. Soc. 69 (1963), 422-433 
[3] Dzenan Gusic, Prime Geodesic Theorem for Compact Riemann Surfaces, international journal of circuits, systems and signal processing, pp. 747-753, Volume 13, 2019

[4] P.Griffiths, J.Harris, Principle of algebraic geometry, Wiley-interscience, New York, 1978.

[5] K. Kodaira Complex Manifolds and Deformations of Complex Structures, Springer, Classics In Mathemtics, 2005.

[6] K. Gawedzki, Lectures on Conformal field theory, in Quantum Fields and String: A Course for mathematicians, Vol 2 , A.M.S

[7] Gromov, M. . "Pseudo holomorphic curves in symplectic manifolds". Inventiones Mathematicae. 82 (2), 1985: 307-347.

[8] D.A. Cox, S. Katz,Mirror symetrie and Algebraic geometry, Mathematical Surveys and Monographs V68, A.M.S 1999.

[9] K.Hori, S. Katz, A. Klemm, R. Pandharipande, R. Thomas, C. Vafa, R. Vakil, E. Zaslow, Mirror Symmetry Clay Mathematics Monographs, Vol 1 A.M.S, 2003.

[10] S.Donaldson, P. Kronheimer, The geometry of four manifolds, Oxford University Press, 1990.

[11] E. Witten,"Topological quantum field theory", Comm. Math. Phys., Volume 117, Number 3 (1988), 353-386 D.A. Cox, S. Katz,Mirror symetrie and Algebraic geometry, Mathematical Surveys and Monographs V68, A.M.S 1999.

[12] M. Kontsevich, Y.Manin, Gromov-Witten Classs, Quantum Cohomology, and enumerative Geometry, Mirror symetry II, A.M.S/IP Studies in advanced Math., Vol 1 A.M.S, 1997, pp. 607-653.

[13] H. Clemens, A Scrapbook of Complex Curve Theory, G.S.M Vol 55, A.M.S Studies in advanced Math., Vol 1 A.M.S, 1997, pp. 607-653.

[14] H. Morrison, Mirror symmetry and rational curves on quintic threefolds: a guide for mathematicians,J. Amer. Math. Soc. 6 (1993) 223-247

[15] A. Polishchuk, E. Zaslow, Categorical Mirror Symmetry: The Elliptic Curve, Adv.Theor.Math.Phys.2:443-470,1998
[16] D.McDuff, D.Salamon, Introduction to symplectic géometry Oxford University Press, 1995.

C. Voisin Symétrie miroir, Panoramas et [17] synthèses Numéro 2, 1996 S.M.F 